\title{
Mutual Coupling Effects on Pattern Diversity Antennas for MIMO Femtocells
}

\author{
Yue Gao, Shihua Wang, Oluyemi Falade, Xiadong Chen, Clive Parini, and Laurie Cuthbert
}

School of Electronic Engineering and Computer Science, Queen Mary, University of London, London E1 4NS, UK

Correspondence should be addressed to Yue Gao, yue.gao@elec.qmul.ac.uk

Received 8 December 2009; Revised 22 February 2010; Accepted 9 April 2010

Academic Editor: Hoi Shun Lui

Copyright () 2010 Yue Gao et al. This is an open access article distributed under the Creative Commons Attribution License, which permits unrestricted use, distribution, and reproduction in any medium, provided the original work is properly cited.

Diversity antennas play an important role in wireless communications. However, mutual coupling between multiple ports of a diversity antenna has significant effects on wireless radio links and channel capacity. In this paper, dual-port pattern diversity antennas for femtocell applications are proposed to cover GSM1800, UMTS, and WLAN frequency bands. The channel capacities of the proposed antennas and two ideal dipoles with different mutual coupling levels are investigated in an indoor environment. The relation between mutual coupling and channel capacity is observed through investigations of these antennas.

\section{Introduction}

Driven by demands from mobile users, there is nowadays a strong requirement to provide sustainable data rates everywhere, not just reception of signals. According to recent surveys, $50 \%$ of mobile phone calls and $70 \%$ of mobile data services will take place indoors in the next few years [1]. Using macrocells to provide coverage is expensive when serving indoor customers who require high data rates and QoS (Quality of Service). FAPs (Femtocell access points) or home-base stations [2] have been proposed in such a scenario to enhance the coverage and data rate. An FAP is a low-power access point that is built using a standard air interface (GSM, UMTS, or LTE), while the backhaul connection makes use of a broadband connection such as optical fibre or DSL (Digital Subscriber Line).

The use of femtocells benefits both users and operators. Users enjoy a better signal quality due to the proximity between transmitter and receiver. Since indoor traffic is transmitted over the IP backhaul, femtocells help the operator to manage the exponential growth of traffic and increase the reliability of macrocell networks. It has been shown from previous studies that a two-tier underlay with 50 femtocells per cell-site network can obtain a nearly 25 times improvement in overall spatial reuse compared to a macrocell-only network [2]. However, the cochannel deployment of such a large femtocell layer will impact existing macrocell networks, affecting their capacity and performance [3]. Femtocell in cochannel operation with an existing macrocellular network is technically more challenging, but is also more rewarding for the operator because of the potentially increased spectral efficiency per area through spatial frequency reuse. Especially in two-tier networks with universal frequency reuse, the near-far effect from cross-tier interference creates dead spots where reliable coverage cannot be guaranteed to users in either tier. Theoretical studies have shown that employing multiple antenna transmission at the macrocell and femtocells provides independent and spatially distinct copies of the transmitted signal, thereby providing increased spatial reuse and increased robustness against near-far effects at the user terminal [4]. In this paper, a dual-port pattern diversity antenna design is proposed for the MIMO (Multiple Input Multiple Output) femtocell application to enhance the earlier theoretical studies. Mutual coupling effects between the ports on the channel capacity are analyzed in an indoor MIMO channel model.

\section{Antenna Structure and Performance}

Microstrip patch antenna is generally chosen as the technology for diversity antennas for both mobile base stations and terminals because of its advantages of compactness 


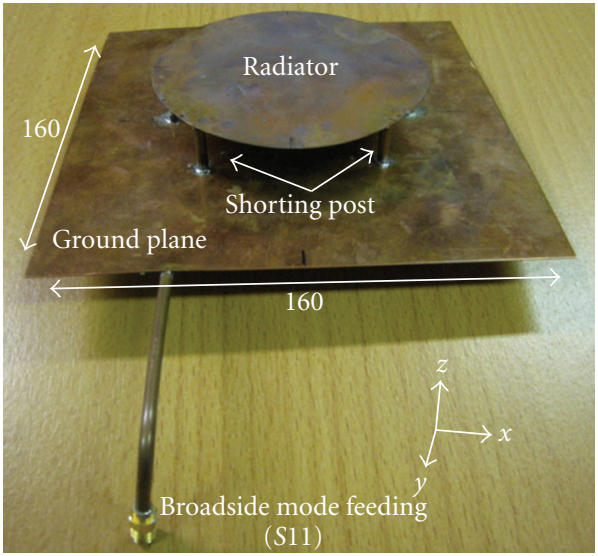

(a) Top view

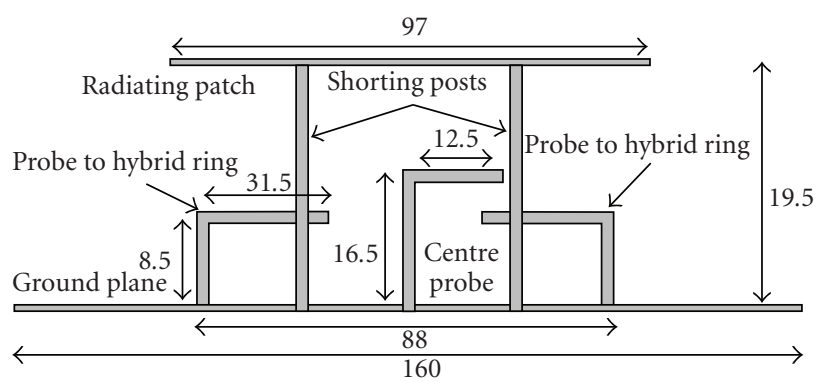

(b) Side view

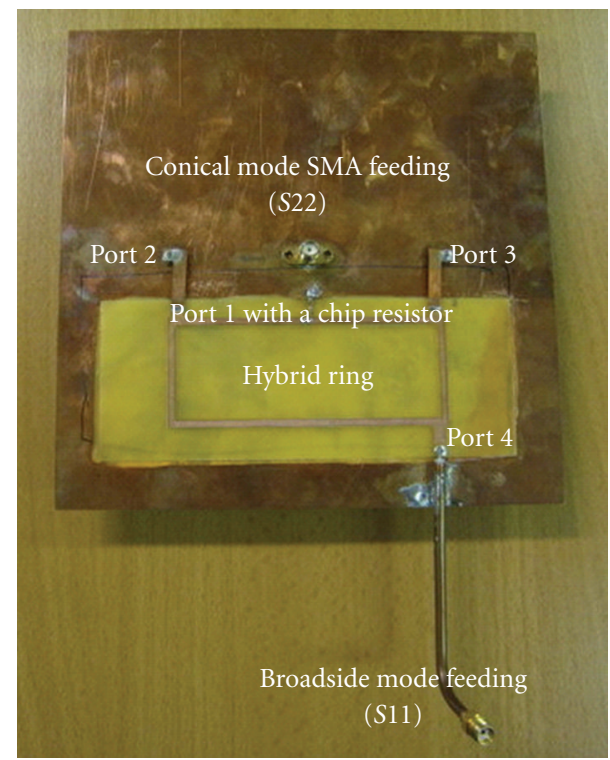

(c) Back view

FIGURE 1: Geometry of the proposed diversity antenna (Design 1): (a) top view, (b) side view, and (c) back view (Units in mm).

and easy fabrication $[5,6]$. This paper presents a modified circular microstrip L-probe wideband patch antenna to achieve diversity operations for FAPs based on a previous study [6]. The circular patch with a diameter of $97 \mathrm{~mm}$ is built on a square ground plane with a size of $160 \mathrm{~mm}$ by $160 \mathrm{~mm}$, as shown in Figure 1(a). The patch is supported by four shorting posts, as shown in Figure 1(b). It is fed by an L-shaped probe for the conical mode and a hybrid ring (e.g., Port 4) for the broadside mode, as shown in Figure 1(c). The hybrid ring is built on an FR4 PCB (Printed Circuit Board) with a substrate thickness of $1.6 \mathrm{~mm}$ and permittivity of 3 . Ports 2 and 3 of the hybrid ring extend two L-probes to the radiator, and Port 1 is terminated by a $50 \Omega$ chip resistor. The detailed dimensions and feeding structure are shown in Figure 1(b).

Computer Aided Design (CAD) was carried out by using the Computer Simulation Technology (CST) Microwave Studio package, which utilizes the Finite Integral Technique
(FIT) for electromagnetic computation [7]. As discussed in [6], the performance of the antenna is dependent on a number of design parameters, such as antenna height, radius of the radiator, shorting posts, and hybrid ring. Based on simulations using CST Microwave studio, a prototype of this antenna has been fabricated in the Antenna Measurement Laboratory at Queen Mary University of London (QMUL).

The $S$-parameters of the antenna were measured by an HP8720ES vector network analyzer and compared to the simulated results in Figure 2. The impedance bandwidth at $-10 \mathrm{~dB}$ for both measured and simulated $S 11$ and $S 22$ operates from $1.7 \mathrm{GHz}$ to $2.3 \mathrm{GHz}$, while at $-6 \mathrm{~dB}$ it operates from $1.68 \mathrm{GHz}$ to $2.5 \mathrm{GHz}$ which covers GSM1800, UMTS, LTE, and WLAN. It can be seen in Figure 2 that measured $S 11$ and $S 22$ have the same number of resonances as those of the simulated values, and both measurement and simulation show similar amount of bandwidth at $-10 \mathrm{~dB}$. However, the main resonance of the measured $S 11$ and $S 22$ are shifted from 


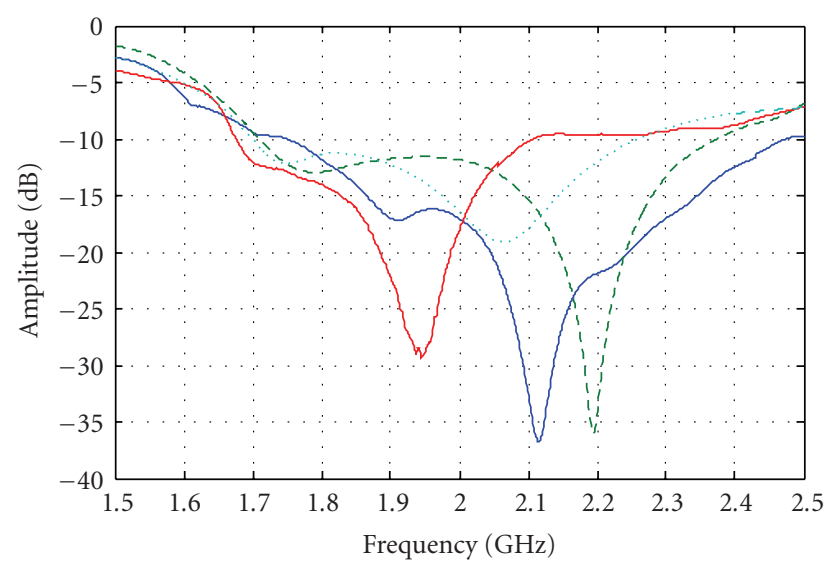

$\begin{array}{lll}- \text { Measured } S 11 & - & \text { Measured S22 } \\ --- \text { Simulated } S 11 & \ldots . & \text { Simulated S22 }\end{array}$

Figure 2: Simulated and measured return losses of the antenna (Design 1).

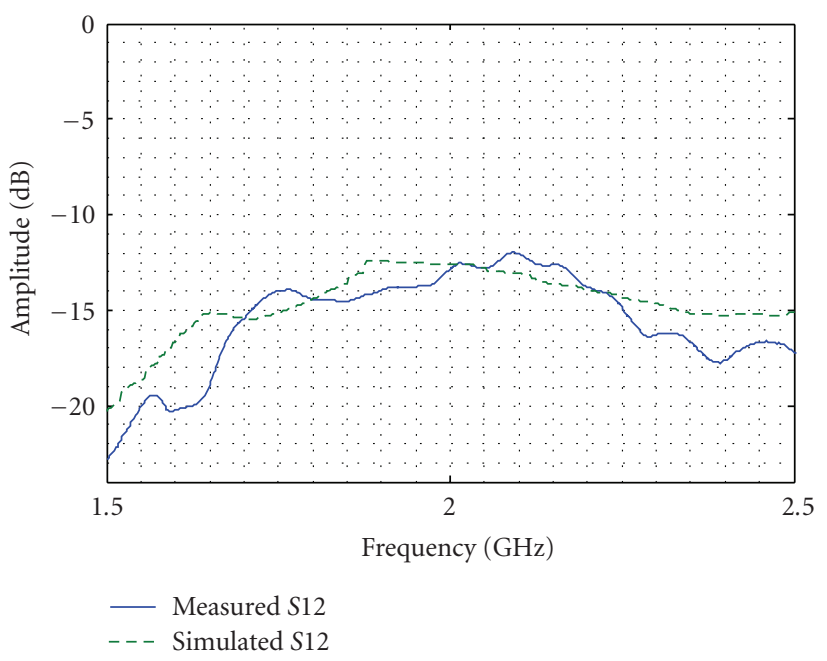

Figure 3: Isolation between the conical and broadside modes.

that of the simulated ones by about $100 \mathrm{MHz}$. The measured $S 22$ is also $10 \mathrm{~dB}$ lower than the one simulated. This variation in the impedance bandwidth is because of tolerances in the soldering and manufacturing of the connector to the hybrid ring port and the long coaxial cable used in the process of measurement.

The isolation of both measurement and simulation is around $12 \mathrm{~dB}$ as shown in Figure 3. The reason for this poor isolation is because the position of the frequency for matching does not always correlate to that for better isolation. It has been shown that total length of the hybrid ring has significant effects on the antenna performance [8]. For instance, when the length of the hybrid ring is exactly $(3 / 2) \lambda_{0}$ (where $\lambda_{0}$ is the wavelength of the first resonance), there is always a wider bandwidth but poorer isolation. The length of the hybrid ring at $(3 / 2) \lambda_{0}$ is adopted in our design, which is referred to as Design 1 in the rest of paper.

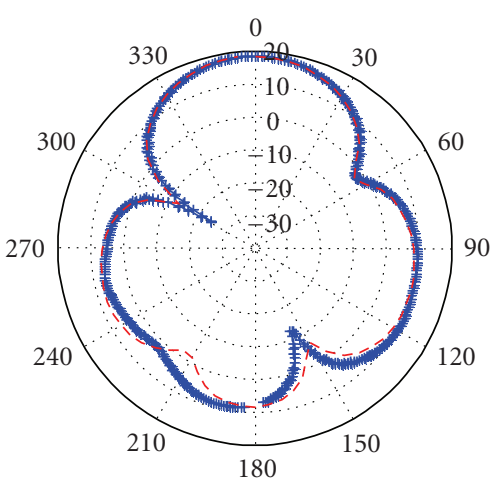

(a)

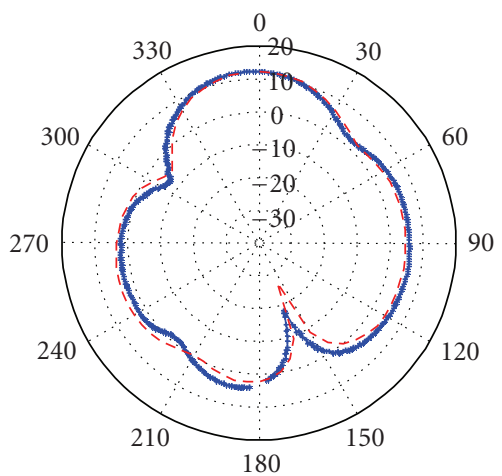

(b)

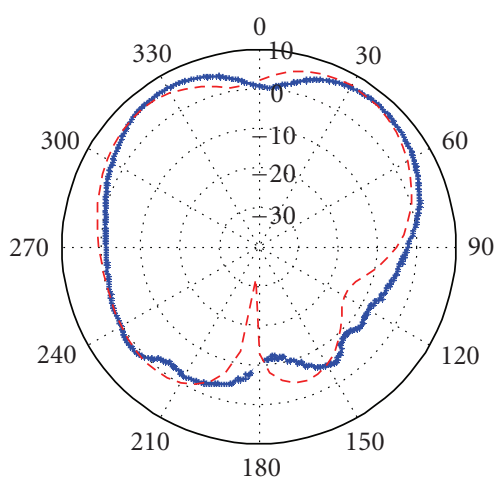

(c)

Figure 4: Measured $(+)$ and simulated $(-)$ copolarized radiation patterns for broadside mode (S11) in XZ-plane at (a) $1.7 \mathrm{GHz}$, (b) $1.85 \mathrm{GHz}$, and (c) $2.1 \mathrm{GHz}$.

The radiation patterns of the antenna were measured in an anechoic chamber in the Antenna Measurement Laboratory at QMUL. The measured and simulated copolarized and cross-polarized radiation patterns in the XZ-plane for broadside mode $(S 11)$ at $1.7 \mathrm{GHz}, 1.85 \mathrm{GHz}$, and $2.1 \mathrm{GHz}$ are plotted in Figures 4 and 5. The measurements agree with the simulations very well. It can be seen that the copolarization is about $15 \mathrm{~dB}$ higher than the cross-polarization. The dominant copolarized radiation patterns in Figure 4 also have $10 \mathrm{~dB}$ stronger radiations in upward directions.

The simulated and measured radiation patterns for the conical mode (S22) at $1.7 \mathrm{GHz}, 1.85 \mathrm{GHz}$, and $2.1 \mathrm{GHz}$ are 


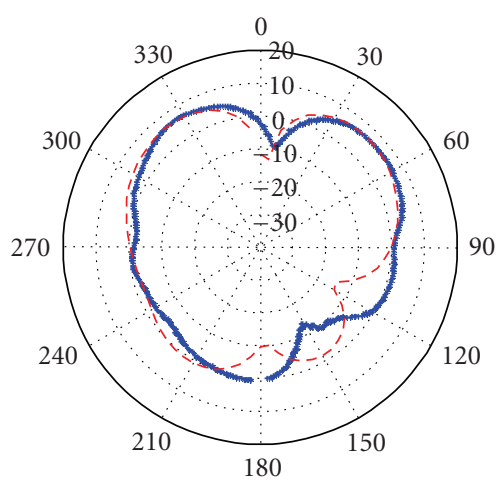

(a)

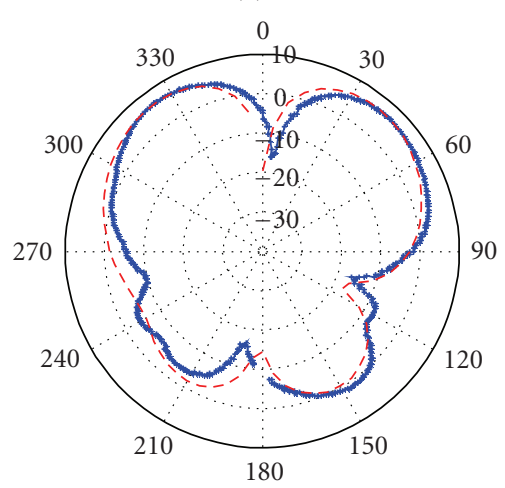

(b)

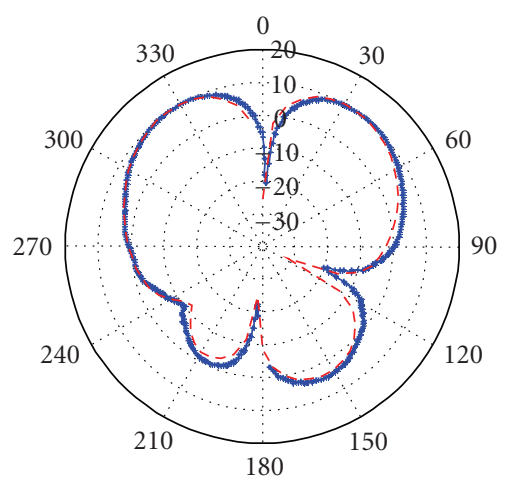

(c)

FIGURE 5: Measured (+) and simulated (-) cross-polarized radiation patterns for broadside mode (S11) in XZ-plane at (a) $1.7 \mathrm{GHz}$, (b) $1.85 \mathrm{GHz}$, and (c) $2.1 \mathrm{GHz}$.

plotted in Figures 6 and 7. The measured and simulated patterns are almost identical. In comparison with the broadside mode, the radiation patterns at conical mode have a similar trend, that is the copolarization is higher than cross-polarization. However, the dominant copolarizations have nulls at the upward direction (0 degree) and stronger radiations at 50 degree elevation angles as shown in Figure 6. These patterns are complementary to those in the broadside mode in Figure 4, which indicates good pattern diversity characteristics.

In the case of multiantenna applications such as a diversity antenna array, it is required that the mutual coupling

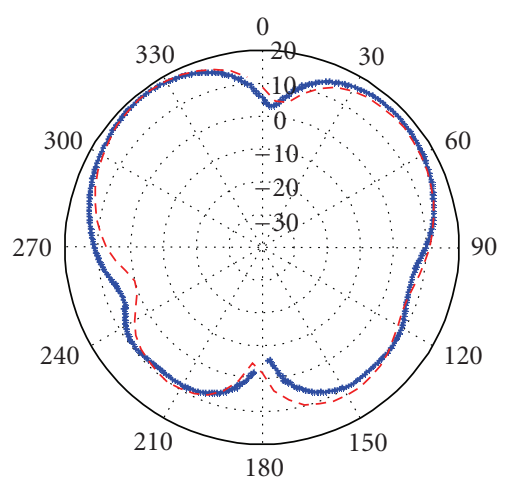

(a)

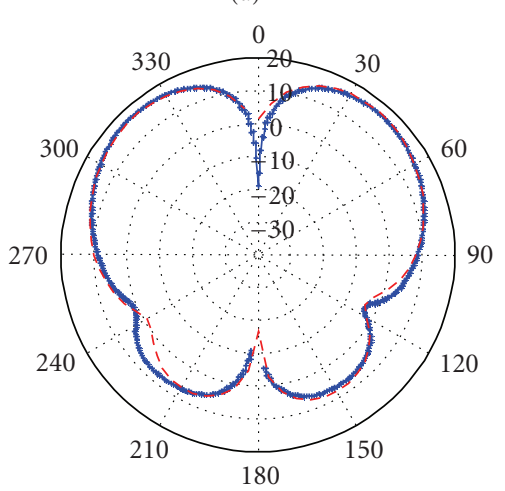

(b)

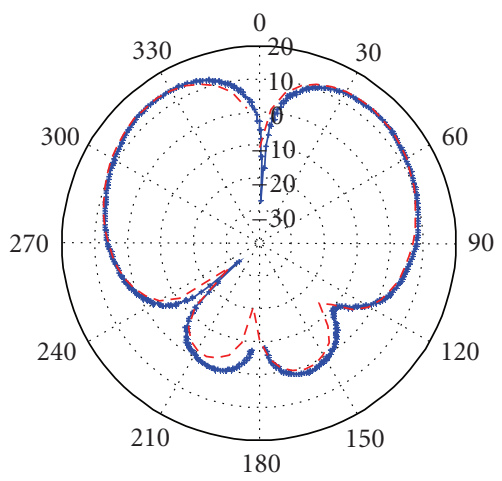

(c)

FIGURE 6: Measured $(+)$ and simulated $(-)$ copolarized radiation patterns for conical mode (S22) in XZ plane at (a) $1.7 \mathrm{GHz}$, (b) $1.85 \mathrm{GHz}$, and (c) $2.1 \mathrm{GHz}$.

between multiple antennas should be minimised to maintain high efficiency of the multiple antennas [9, 10]. Further investigations were conducted using CST Microwave Studio to minimise the mutual coupling between the broadside and conical modes. As discussed earlier, the length of the hybrid ring has significant effects on the antenna performance, and there is tradeoff between the bandwidth and isolation. Design 1 with the hybrid ring length of $(3 / 2) \lambda_{0}$ achieved a wider bandwidth but with a $12 \mathrm{~dB}$ isolation; by tuning the length of the hybrid ring to $2 \lambda_{0}$, an isolation better than $30 \mathrm{~dB}$ over the frequency bandwidth is achieved, as shown in Figure 8. However, the impedance bandwidth is narrower 


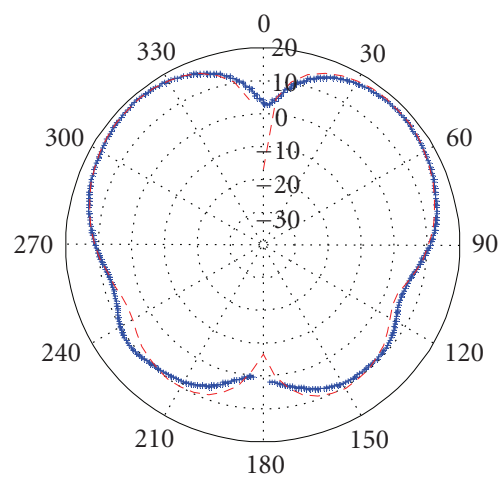

(a)

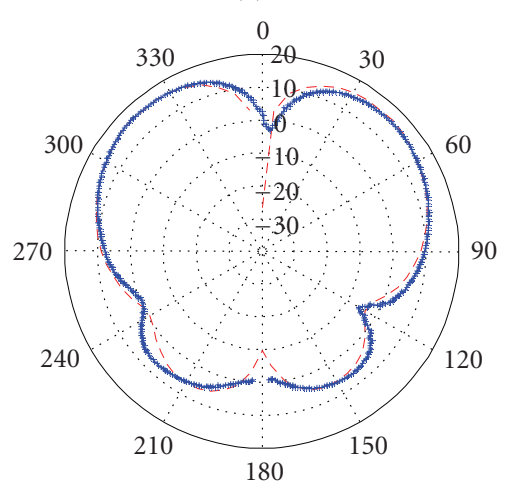

(b)

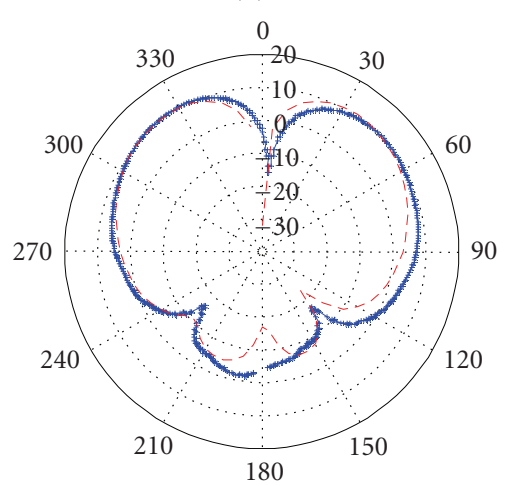

(c)

FIGURE 7: Measured $(+)$ and simulated $(-)$ cross-polarized radiation patterns for conical mode (S22) in XZ plane at (a) $1.7 \mathrm{GHz}$, (b) $1.85 \mathrm{GHz}$, and (c) $2.1 \mathrm{GHz}$.

than that of Design 1. The second model is referred to as Design 2. The dimensions of the hybrid ring are shown in Figure 9.

\section{Mutual Coupling Effects on Channel Capacity}

A ray-tracing-based channel model developed at QMUL [11] was revised to evaluate MIMO channel performance for a femtocell scenario at the $2.1 \mathrm{GHz}$ band. As shown in Figure 10, the model is based on the second floor of the Electronic Engineering department at QMUL in which the building structure can be treated as a typical femtocell environment, for example, offices or home.

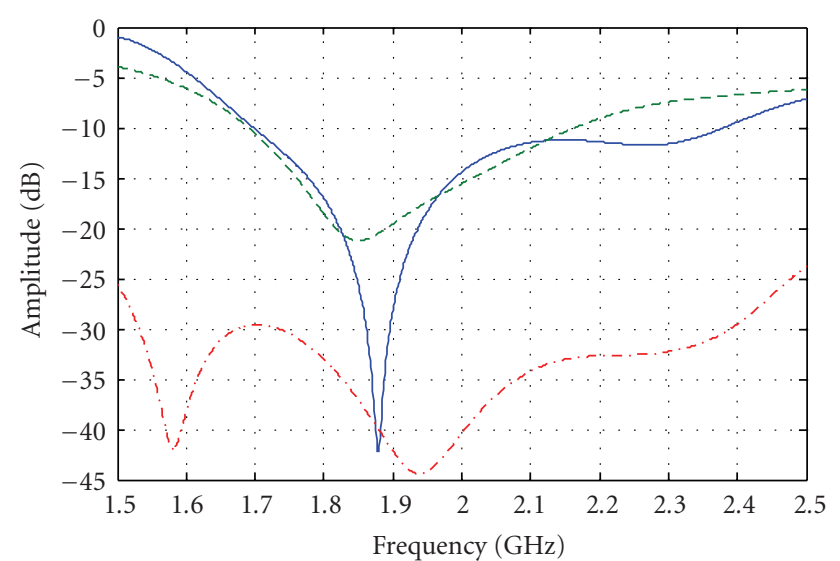

FIgURE 8: S-parameters for the Design 2.

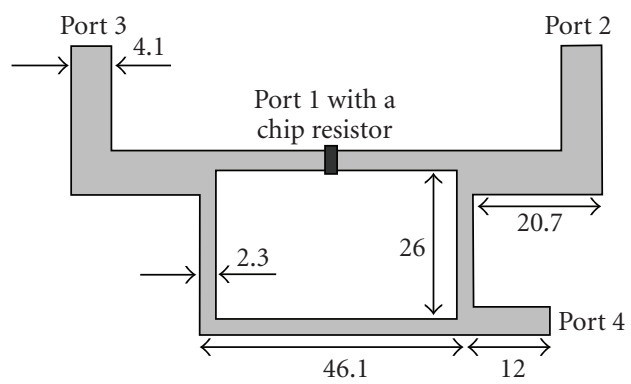

Figure 9: The geometry of the hybrid ring for better isolation in Design 2.

In this model, there are three different materials that have been used to model the real environment: wood, glass, and concrete wall. All these materials are assumed to be homogenous and their material properties at $2.1 \mathrm{GHz}$ are summarized in Table 1 [12].

Different types of antennas are evaluated for the FAP in the environment of Figure 10. These antennas are (i) dual ideal dipoles separated by $\lambda / 2$ ( $\lambda$ is the free space wavelength at $2.1 \mathrm{GHz}$ ), (ii) Design 1, and (iii) Design 2. Their simulated 3D radiation patterns, which are affected by the mutual coupling levels, have been imported into the model to evaluate the channel capacity. These three types of antennas and their mutual coupling level are summarized in Table 2.

In the femtocell scenario, the bandwidth and the transmitted power for the transmitter are set to be $5 \mathrm{MHz}$ and $20 \mathrm{dBm}$, respectively [13]. The FAP is placed $3.3 \mathrm{~m}$ high along the corridor to represent a mounted access point. Several receivers are randomly placed in different rooms, as indicated in Figure 10. Each receiver consists of two omnidirectional antennas with $\lambda / 4$ spacing at the normal desk height. It is assumed that the channel is unknown to the FAP and signal to noise ratio (SNR) is $10 \mathrm{~dB}$ for all the cases. Mutual coupling effects for these antennas are hence evaluated in terms of channel capacity. 


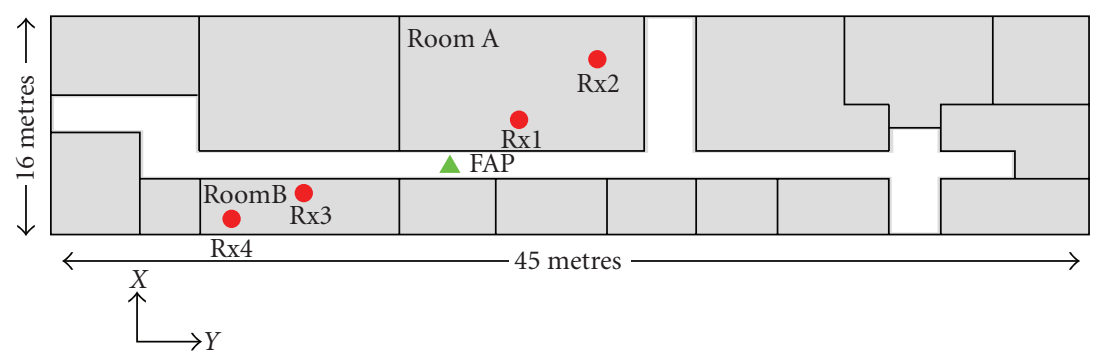
Receivers
Corridor
$\triangle$ FAP
Offices and labs

FIGURE 10: Floor plan of the second floor of Electronic Engineering Building at QMUL.

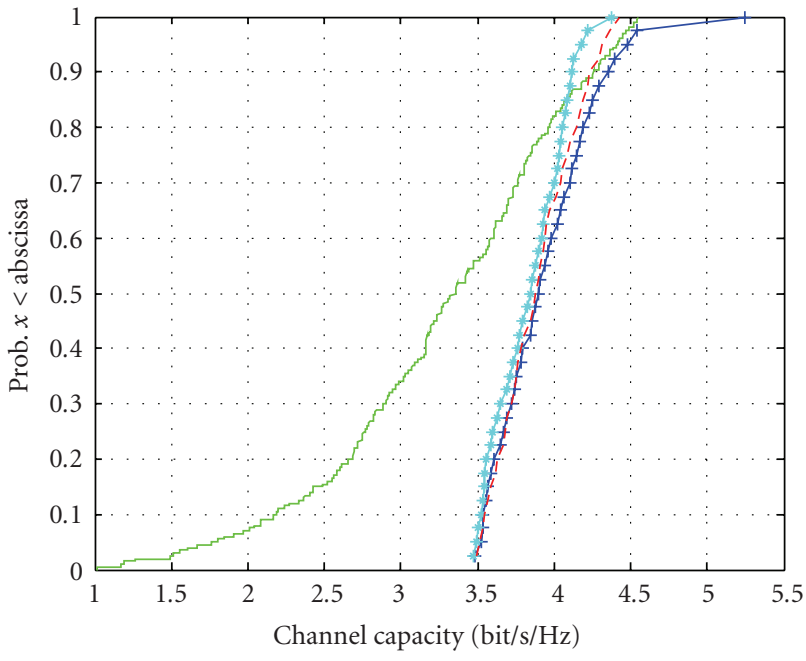

(a)

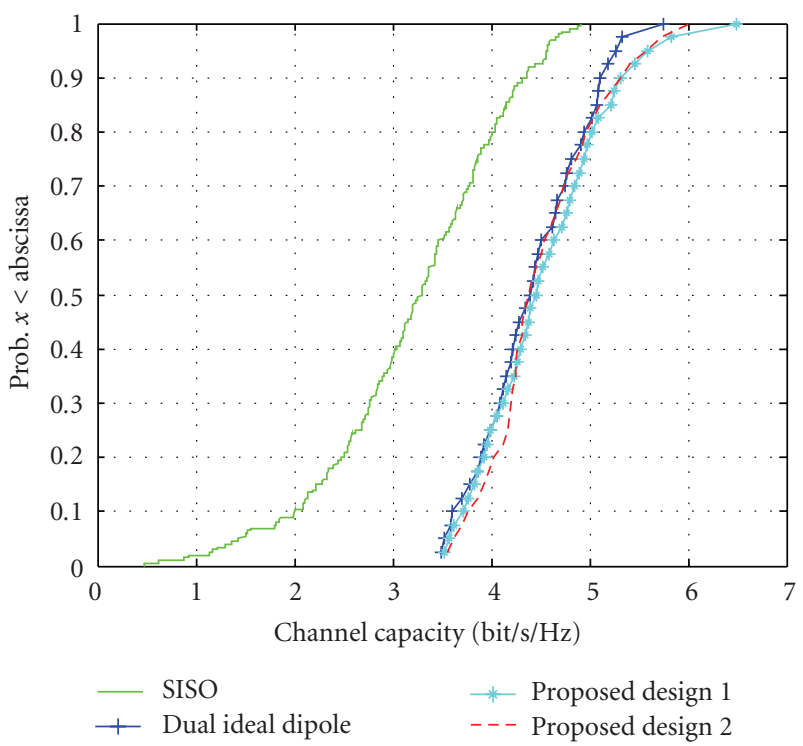

(b)

Figure 11: CDFs for different receiver locations at Room A: (a) Rx1 and (b) Rx2.

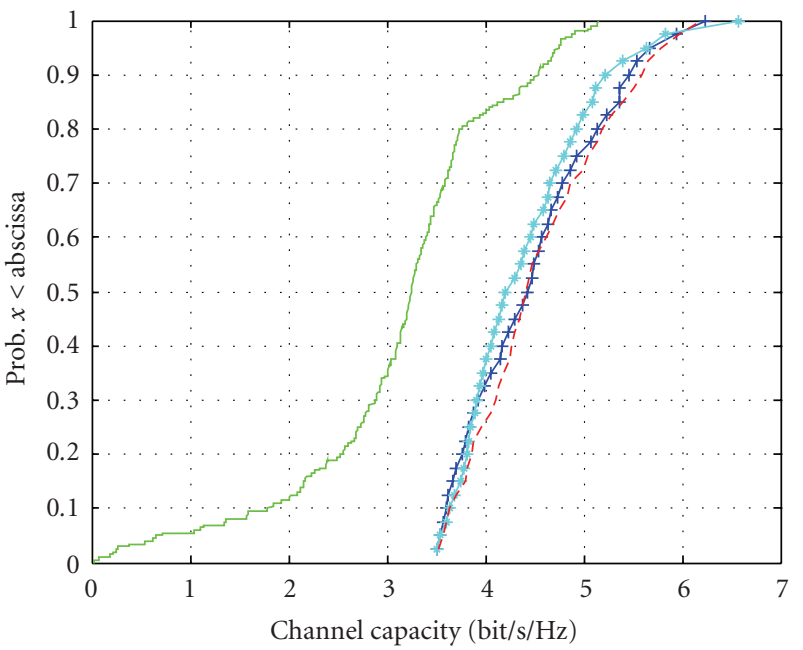

(a)

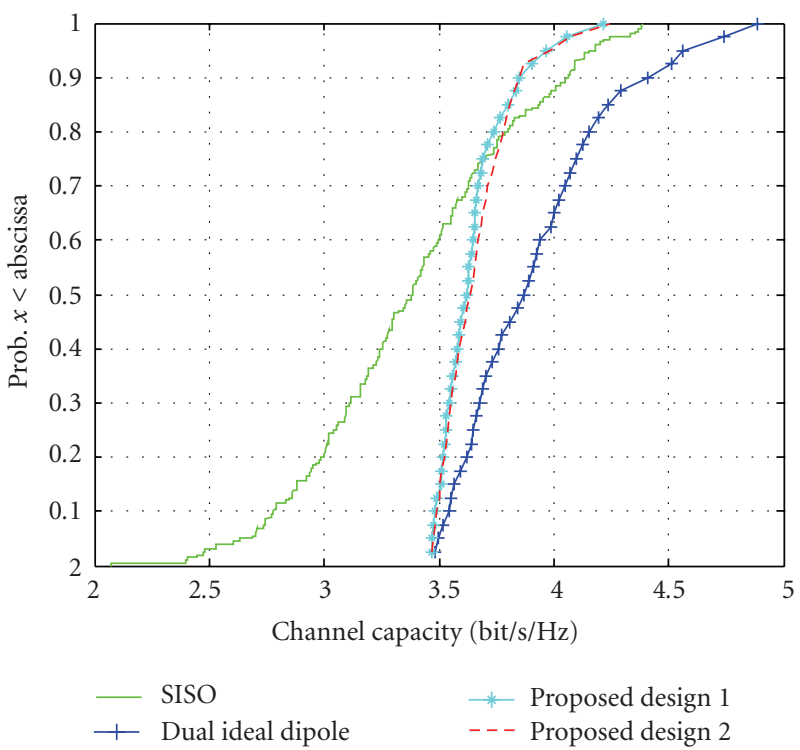

(b)

Figure 12: CDF for different receiver locations at Room B: (a) Rx3 and (b) $\mathrm{Rx} 4$. 
TABLE 1: Material properties used at 2.1 GHz.

\begin{tabular}{lccc}
\hline Material & Permittivity & Conductivity & Thickness \\
\hline Concrete (wall, & 5.820 & 0.0596 & $0.3 \mathrm{~m}$ \\
floor, and ceiling) & & 0.0033 & $0.05 \mathrm{~m}$ \\
Wood (door) & 2.5 & 0.00278 & $0.005 \mathrm{~m}$ \\
\hline
\end{tabular}

TABLE 2: Mutual coupling levels of the antennas.

\begin{tabular}{lc}
\hline Antenna Types for FAP & Mutual coupling levels \\
\hline (i) Dual ideal dipole antennas & No coupling \\
(ii) Proposed antenna design 1 & $-12 \mathrm{~dB}$ coupling \\
(iii) Proposed antenna design 2 & $-30 \mathrm{~dB}$ coupling \\
\hline
\end{tabular}

A $2 \times 2$ MIMO system's capacity can be calculated as [14]

$$
C(\rho)=\log _{2} \operatorname{det}\left[I_{n}+\frac{\rho}{n} H H^{\dagger}\right]
$$

where $\mathbf{H}$ is the normalized $n \times n$ channel matrix, $I$ is the identity matrix and $\rho$ is the SNR. With a narrowband assumption, the channel response $H$ is given by [15]

$$
h_{i j}=\sum_{k=0}^{M} \sqrt{P_{k}} \cdot e^{j \theta_{k}} \cdot e^{j 2 \pi f_{0} \tau_{k}},
$$

where $P_{k}, \theta_{k}$, and $\tau_{k}$ are the received power, phase, and time delay of the $k$ th ray, respectively. $M$ is the total number of rays. So that for a $2 \times 2 \mathrm{MIMO}$ case, channel realization the $\mathrm{H}$ matrix can be built as

$$
H=\left[\begin{array}{ll}
h_{1,1} & h_{1,2} \\
h_{2,1} & h_{2,2}
\end{array}\right] .
$$

Each of $h_{i, j}$ can be calculated by using (2). It has been assumed that receivers $(\mathrm{Rx})$ move along the $y$-axis as shown in Figure 10, so that all of the spatial fading possibilities are sampled across the area. The cumulative distribution function (CDF) plots for different receiver locations at Room $\mathrm{A}$ and Room B are shown in Figures 11 and 12, respectively.

It has been observed that our proposed designs with mutual coupling effect slightly degrade channel capacity for all the cases in Room A and Room B, compared with the dual ideal dipoles which do not take into account the mutual coupling effect as shown in Figures 11 and 12. For the receivers $\mathrm{Rx} 3$, Design 2 with $-30 \mathrm{~dB}$ mutual coupling performs slightly better than Design 1 with $-12 \mathrm{~dB}$ mutual coupling. However, for the receivers $\mathrm{Rx} 1, \mathrm{Rx} 2$, and $\mathrm{Rx} 4$, Design 1 and 2 achieve almost the same capacity, which means that mutual coupling has little effects on the channel capacity when it is smaller than $-12 \mathrm{~dB}$ in this case.

It is noticed in Figure 12(b) that the degradation of the channel capacity with both Design 1 and 2 is much stronger at the receiver $\mathrm{Rx} 4$ than any other receiving locations. The main reason for this phenomenon is because the link between the FAP and $\mathrm{Rx} 4$ is the longest so that propagation between the two ends experiences more scattering. Therefore, the transmitted power arrives with nearly a 360 degree pattern, as seen by the receivers, and the dipole-like radiation pattern is more suitable for such a propagation environment.

\section{Conclusions}

Two pattern diversity antennas operating from $1.68 \mathrm{GHz}$ to $2.5 \mathrm{GHz}$ for femtocell access points have been designed with mutual coupling of $-12 \mathrm{~dB}$ and $-30 \mathrm{~dB}$, respectively. The channel capacity of each of these diversity antennas is evaluated in an indoor MIMO channel environment and compared with that of dual ideal dipoles with no mutual coupling effect. It has been shown that the channel capacity of the proposed Design 2 with very low mutual coupling $(-30 \mathrm{~dB})$ is close to that of an ideal dipole array without mutual coupling in most cases. The exception case from the results shows that the channel capacity not only depends on the mutual coupling levels but also on the propagation environment. The channel capacity of the proposed Design 1 with a mutual coupling of $-12 \mathrm{~dB}$ achieved the similar capacity as that of Design 2. Therefore, it can be concluded that a mutual coupling of $-12 \mathrm{~dB}$ is a reasonable level to maintain a good channel performance, and there is no need to obtain a very low mutual coupling in Design 2 at the expense of the diversity antenna performance, such as impendence bandwidth and compactness.

\section{References}

[1] G. Mansfield, "Femtocells in the US Market—business drivers and consumer propositions," in FemtoCells Europe, ATT, June 2008.

[2] V. Chandrasekhar, J. G. Andrews, and A. Gatherer, "Femtocell networks: a survey," IEEE Communications Magazine, vol. 46, no. 9, pp. 59-67, 2008.

[3] H. Claussen, "Performance of macro- and co-channel femtocells in a hierarchical cell structure," in Proceedings of 18th Annual IEEE International Symposium on Personal, Indoor and Mobile Radio Communications (PIMRC '07), Athens, Greece, September 2007.

[4] V. Chandrasekhar, M. Kountouris, and J. G. Andrews, "Coverage in multi-antenna two-tier networks," IEEE Transactions on Wireless Communications, vol. 8, no. 10, pp. 5314-5327, 2009.

[5] Y. Gao, X. Chen, Z. Ying, and C. G. Parini, "Design and performance investigation of a dual-element PIFA array at 2.5 GHz for MIMO terminal," IEEE Transactions on Antennas and Propagation, vol. 55, no. 12, pp. 3433-3441, 2007.

[6] S.-L. S. Yang and K.-M. Luk, "Design of a wide-band Lprobe patch antenna for pattern reconfiguration or diversity applications," IEEE Transactions on Antennas and Propagation, vol. 54, no. 2, part 1, pp. 433-438, 2006.

[7] “CST-Microwave Studio,” 2009, User's Manual.

[8] J. Olszewska, S. Vaccaro, J.-F. Zürcher, and A. K. Skrivervik, “A new hybrid-ring geometry well suited for CAD implementation," Microwave and Optical Technology Letters, vol. 40, no. 4, pp. 285-287, 2004.

[9] Z. Ying and D. Zhang, "Study of the mutual coupling, correlations and efficiency of two PIFA antennas on a small ground plane," in Proceedings of IEEE Antennas and Propagation Society International Symposium, vol. 3, pp. 305308, Washington, DC, USA, July 2005.

[10] Y. Gao, X. Chen, and C. G. Parini, "Channel capacity of dual-element modified PIFA array on small mobile terminal," Electronics Letters, vol. 43, no. 20, pp. 1060-1062, 2007. 
[11] S. Wang, X. Chen, and C. G. Parini, "Ray-tracing based channel model for $5 \mathrm{GHz}$ WLAN," in IEEE Antennas and Propagation Society International Symposium, pp. 1-4, Charleston, SC, USA, 2009.

[12] Ofcom Final Report on, "Dielectric measurements of typical materials used in the construction of buildings and FSS structures work package 4," Tech. Rep., March 2004.

[13] A. Dow, "Evolution to Femto Cells for Radio Access," AlcatelLucent, September 2008.

[14] G. Foschini and M. Ganst, "On limits of wireless communications in a fading environment when using multiple antennas," Wireless Personal Communications, vol. 6, no. 3, pp. 311-335, 1998.

[15] C.-N. Chuah, G. Foschini, R. A. Valenzuela, D. Chizhik, J. Ling, and J. Kahn, "Capacity growth of multi-element arrays in indoor and outdoor wireless channels," in Proceedings of IEEE Wireless Communications and Networking Conference (WCNC'00), vol. 3, pp. 23-28, September 2000. 

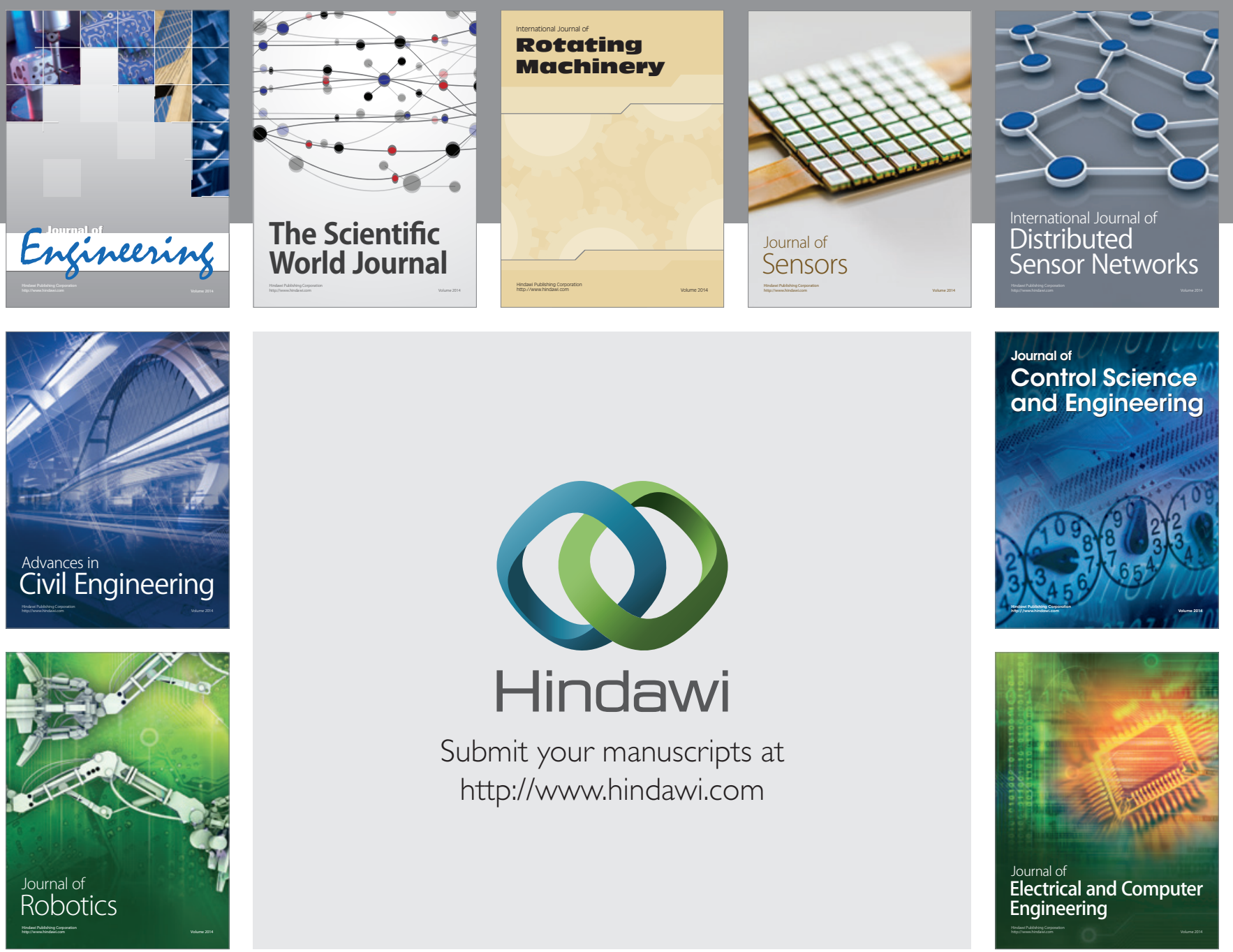

Submit your manuscripts at

http://www.hindawi.com
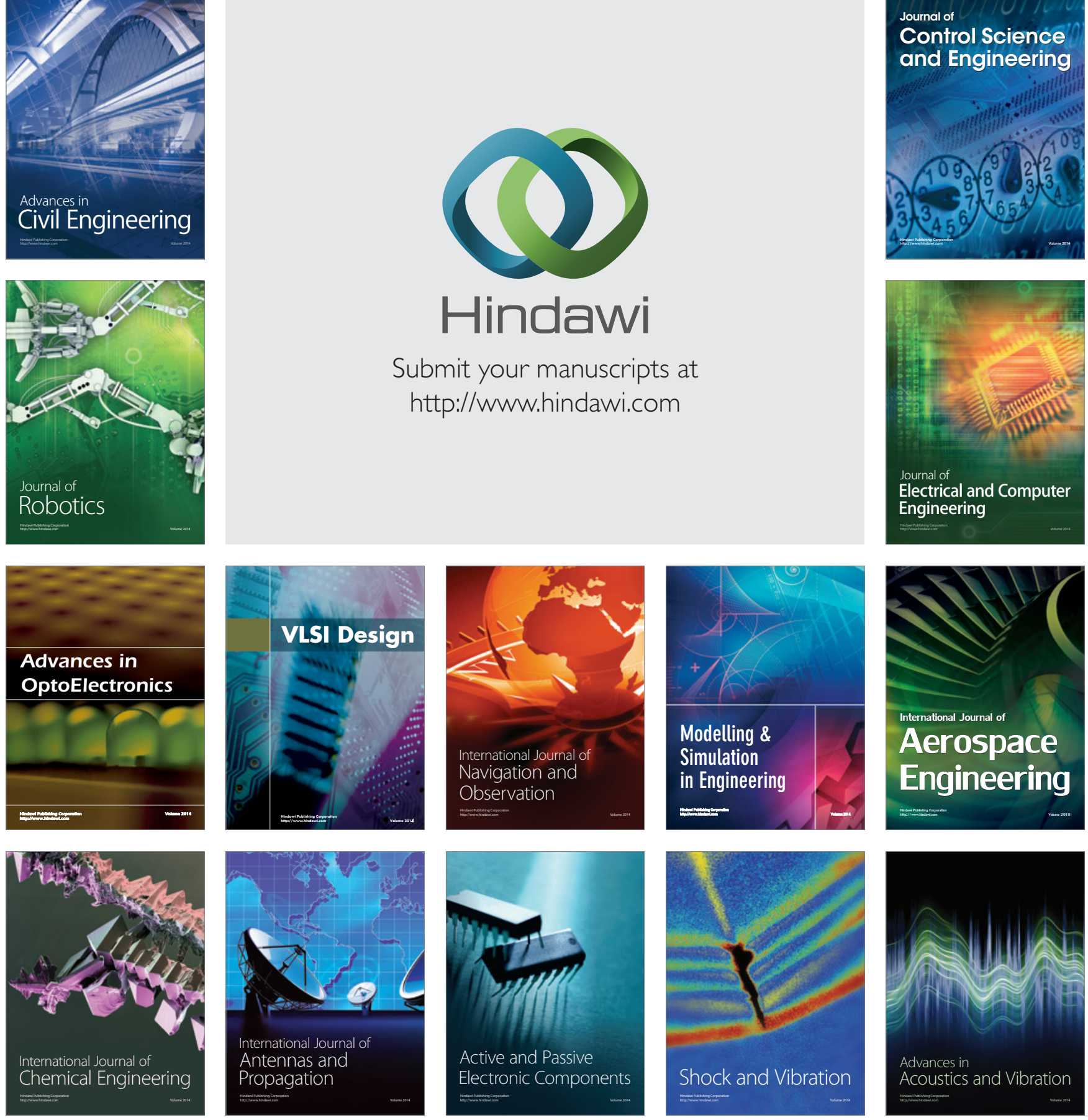